\title{
ANSIEDADE DOS ENFERMEIROS DA ESTRATÉGIA SAÚDE DA FAMÍLIA
}

\author{
ANXIETY OF NURSES OF THE FAMILY HEALTH STRATEGY
}

\author{
Juliana Sangaletti ${ }^{2}$ \\ Luciane B.Ceretta ${ }^{3}$ \\ Maria Tereza Soratto ${ }^{4}$ \\ Recebido em: 05 jun. 2016 \\ Aceito em: 13 jun. 2018
}

RESUMO: Estudo com objetivo de identificar os fatores associados à Ansiedade do Enfermeiro da Estratégia Saúde da Família (ESF). Pesquisa de abordagem qualiquantitativa, descritiva, exploratória e de campo. O estudo foi desenvolvido com 07 enfermeiros que trabalham em uma Estratégia Saúde da Família de Santa Catarina. Aplicou-se a Escala de Avaliação de Ansiedade de Hamilton e questionário semiestruturado sobre os fatores relacionados à ansiedade dos Enfermeiros. A análise e interpretação dos dados qualitativos foram organizadas a partir da análise de conteúdo com a categorização dos dados, através da ordenação, classificação e análise final dos dados pesquisados. Na fase de análise de dados quantitativos, as informações foram quantificadas através do Microsoft Office Excel e apresentados por meio de análise quantitativa. Os resultados da pesquisa realizada identificaram que $85,72 \%$ dos enfermeiros apresentaram nível de ansiedade leve e 14,28\% nível moderado. Os fatores associados à ocorrência de ansiedade nos enfermeiros da ESF vincularam-se principalmente ao excesso de trabalho, seguido da falta de valorização profissional; pressão exercida no processo de trabalho; preocupação e agitação no cotidiano do trabalho. Sendo assim, sugere-se um aprofundamento da pesquisa em relação aos fatores determinantes da ansiedade e as formas de melhor combatê-la no cotidiano do exercício da profissão.

Palavras-chave: Ansiedade. Enfermeiro. Saúde do Trabalhador. Estratégia Saúde da Família.

ABSTRACT: Study aiming to identify the factors associated with the Anxiety of nurses of the family health strategy. Research of qualitative and quantitative approach, descriptive, exploratory and of field. The study was carried out with 07 nurses who work in a family health Strategy. Applied to anxiety rating scale of Hamilton and semistructured questionnaire on related factors to anxiety of nurses. The analysis and interpretation of qualitative data were organized from the content analysis with the categorization of the data, by ordering, sorting, and final analysis of the data searched. In the analysis phase of quantitative data, the information was quantified through the Microsoft Office Excel and presented by means of quantitative analysis. The results of the survey have identified that $85.72 \%$ of nurses showed mild anxiety level and $14.28 \%$ moderate level. The factors associated with the occurrence of anxiety in nurses of the

\footnotetext{
${ }^{1}$ Artigo baseado na Monografia Pós Graduação em Especialização em Gestão da Atenção Básica de SaúdeUNESC

2 Enfermeira. Especialista em Gestão da Atenção Básica em Saúde - UNESC - Universidade do Extremo Sul de Santa Catarinense. Email: j.sangaletti@hotmail.com.

${ }^{3}$ Enfermeira. Doutora em Ciências da Saúde, Mestrado em Enfermagem - UNESC - Universidade do Extremo Sul de Santa Catarina - Criciúma - SC - Brasil. Email: luk@unesc.net.

${ }^{4}$ Enfermeira. Mestre em Educação. Professora do Curso de Pós Graduação Especialização em Gestão da Atenção Básica de Saúde - UNESC - Universidade do Extremo Sul de Santa Catarina - Criciúma - SC - Brasil. E-mail: guiga@unesc.net.
} 
family health strategy linked mainly to overwork, followed by lack of professional valuation; pressure in the process of work; concern and agitation in the daily work. Therefore, it is suggested a deepening of research in relation to determining factors of anxiety and ways to better combat it in the everyday life of the practice of the profession.

Keywords: Anxiety. Nurses. Occupational Health. Family Health Strategy.

\section{INTRODUÇÃO}

"Os problemas de saúde mental estão entre os mais prevalentes em trabalhadores de saúde" (DILELIO et al, 2012, p.512). Os trabalhadores da atenção básica apresentam elevadas prevalências de problemas de saúde, tais como depressão, ansiedade, insônia, fadiga, irritabilidade, esquecimento, dificuldade de concentração e queixas somáticas (DILÉLIO, 2012).

O processo de trabalho em saúde é visto como um universo de saberes e relações humanas, o qual não transforma somente o corpo do sujeito, mas sim, a sua expressão biopsíquica. Por conseguinte, faz-se necessário que o trabalhador perceba seu processo de trabalho, a fim de apreender como se constroem socialmente as necessidades dos usuários dos serviços de saúde e as maneiras de satisfazê-las, recuperando a autonomia dos usuários em seu saber/fazer (OLIVEIRA, 2006). A partir desta definição, observa-se a responsabilidade e o compromisso do cuidar em enfermagem, surgindo o peso da função que poderá desencadear ansiedade e adoecimento do profissional.

A ansiedade pode ser definida como uma condição orientada para o futuro, caracterizada por: apreensão relativa à percepção de não poder controlar ou prever eventos potencialmente aversivos, sintomas corporais de tensão física e desvio do foco de atenção para esses eventos potencialmente aversivos ou às respostas afetivas eliciadas por eles (DESOUSA et al, 2013).

Para ansiedade se define da seguinte forma:

[...] é uma experiência universal humana e é definida como um sentimento persistente de medo, apreensão e desastre iminente, ou tensão e inquietação. De maneira geral, a ansiedade é tida como uma resposta fisiológica natural, mas pode estar relacionada a certas doenças, dentre elas a asma, disfunções gastrintestinais e doença da artéria coronária. Isso torna a ansiedade um alvo importante de estudos, já que essas doenças apresentam alta incidência na população geral (NEVES NETO, 2005, p. 95).

A ansiedade ou síndrome do pensamento acelerado é considerada o mal do século, sem dúvida predomina em quase toda população. Conforme a Organização Mundial de Saúde, $80 \%$ dos indivíduos desenvolverão a ansiedade em algum momento de suas vidas, independente de gênero, idade ou profissão. A mesma é facilmente percebida através do ritmo acelerado na construção de pensamentos e consequentemente nas atitudes cotidianas estressantes, desencadeando uma implicação na saúde emocional (CURY, 2014). 
Em decorrência dessa consideração, como enfermeira atuante na ESF, pôde-se constatar a ansiedade dos profissionais enfermeiros em relação ao processo de trabalho na Atenção Básica e principalmente na Gestão de Pessoas. Os enfermeiros apresentam queixas relacionadas a sintomas cognitivos, somáticos e motores, tornando-se causa de afastamento por doença relacionada ao trabalho. Diante destas questões surgiram inquietações frente à temática: Qual o nível de ansiedade dos enfermeiros? Quais os fatores causais da ansiedade? A gestão do enfermeiro no processo de trabalho na ESF pode estar relacionada à ansiedade?. Quando não controlada adequadamente, a ansiedade no profissional pode vir a ser considerada uma doença profissional.

Pesquisas que abordam a saúde do profissional que atua na atenção básica podem contribuir para discussão das questões de saúde no trabalho da enfermagem, pois a identificação de situações geradoras de sofrimento propicia o desencadeamento de reflexões sobre a forma como o trabalho vem sendo organizado(KESSLER; KRUG, 2012).

Ressalta-se, portanto, a importância de um olhar humanizado e atencioso para a saúde do trabalhador da saúde por parte das instituições de trabalho, assim como, medidas centradas em mudanças na organização do trabalho, com o objetivo de promover o bemestar e evitar o adoecimento desse trabalhador (KESSLER; KRUG, 2012, p.55)

Assim, debater sobre o cuidado, suas formas de ser e agir, é primordial para desenvolver nos profissionais de saúde, a necessidade de se autoconhecer e se reconhecer, a fim de valorizar o cuidar de si para poder cuidar do outro (SANTOS; RADUNZ, 2011).

Desta forma entende-se que estudar a manifestação da ansiedade dos enfermeiros permite compreender e elucidar alguns problemas, tais como a insatisfação profissional, a produtividade do trabalho, o absenteísmo, os acidentes de trabalho e algumas outras doenças ocupacionais, além de permitir a proposição de intervenções e busca de soluções.

A avaliação da ansiedade dos enfermeiros pode oferecer "subsídios para melhorar o processo de trabalho em saúde, a prática clínica, a relação profissional-usuário e orientar a (re)definição de políticas públicas específicas" voltadas para a saúde do trabalhador da atenção básica(FERNANDES et al, 2012, p.405).

Considera-se que são vários os fatores associados à ansiedade do enfermeiro que atua na ESF estando relacionada à: processo de trabalho e sobrecarga de trabalho; gestão de pessoas e da equipe multiprofissional; burocracia e papel administrativo; relacionamento interpessoal com a equipe multiprofissional; acessibilidade ao local de trabalho, sendo que algumas ESF são localizadas na zona rural; instabilidade empregatícia devido às relações políticas.

A melhoria das condições de vida e de trabalho pode gerar "um impacto positivo na saúde, tanto dos enfermeiros quanto da população por eles assistida"(FERNANDES et al, 2012, p.405).

Nesta perspectiva este estudo teve por objetivo identificar o nível de ansiedade dos enfermeiros da Estratégia Saúde da Família (ESF); os fatores associados à ansiedade e as 
estratégias de enfrentamento utilizadas pelos enfermeiros.

\section{MATERIAL E MÉTODOS}

Pesquisa de abordagem quali-quantitativa, descritiva, exploratória e de campo. $\mathrm{O}$ estudo foi desenvolvido com 07 enfermeiros que trabalham na Estratégia Saúde da Família de um município do extremo sul de Santa Catarina. Aplicou-se a Escala de Avaliação de Ansiedade de Hamilton e questionário semi-estruturado sobre os fatores relacionados à ansiedade dos enfermeiros. A análise e interpretação dos dados qualitativos foram organizadas a partir da análise de conteúdo com a categorização dos dados, através da ordenação, classificação e análise final dos dados pesquisados. $\mathrm{Na}$ fase de análise de dados quantitativos, as informações foram quantificadas através do Microsoft Office Excel e apresentados por meio de análise quantitativa.

\section{ESCALA DE ANSIEDADE DE HAMILTON}

A Escala de Ansiedade de Hamilton (1959) compreende 14 itens distribuídos em dois grupos, sendo o primeiro grupo, com 7 itens, relacionado a sintomas de humor ansioso e o segundo grupo, também com 7 itens, relacionado a sintomas físicos de ansiedade - o que possibilita obter escores parciais, ou seja, separadamente para cada grupo de itens.

Segundo Bandeira (2010) dentre as escalas de humor utilizadas mundialmente e que foram traduzidas e adaptadas para a realidade brasileira para o quesito "ansiedade", está a escala de Hamilton-A, que apresenta fácil aplicabilidade (HAMILTON, 1959) e confiabilidade.

O escore total é obtido pela soma dos valores (graus) atribuídos em todos os 14 itens da escala, cujo resultado varia de 0 a 56. Os Graus de Ansiedade segundo Escala de Ansiedade de Hamilton são classificados em: nenhum = 0; leve = 1; médio = 2; forte = 3; máximo $=4$. A soma dos escores obtidos em cada item resulta em um escore total, que varia de 0 a 56. Esse escore deve ser classificado de acordo com os intervalos: 0 (zero) caracteriza ausência de ansiedade; 1 a 17 pontos caracterizam ansiedade leve; 18 a 24 pontos caracterizam ansiedade moderada; 25 a 56 pontos caracterizam ansiedade severa ou intensa.

Para preservar o sigilo dos dados, de acordo com as diretrizes e normas regulamentadoras da Resolução 466/12 que envolve a pesquisa com Seres Humanos, utilizou-se a letra "E" para os enfermeiros entrevistados; seguido do respectivo número E1 a E7. O projeto foi aprovado pelo Comitê de Ética em Pesquisa - Projeto UNESC no 1.305.630. 


\section{RESULTADOS E DISCUSSÃO}

Todos os profissionais apresentaram nível de ansiedade, segundo a Escala de avaliação de ansiedade de Hamilton, sendo classificados em: 85,72\% nível leve e 14,28\%\% nível moderado.

\begin{tabular}{l|l|l|l|l}
$\begin{array}{l}\text { Tabela no 1 - Nível de Ansiedade dos Enfermeiros segundo Escala de avaliação de ansiedade de Hamilton } \\
\text { Nível de Ansiedade }\end{array}$ & No & $\%$ & Enfermeiros (E1 a E7) $\mathbf{n = 0 7}$ \\
\hline Ausência de ansiedade & 0 & 0 & - \\
\hline Ansiedade leve & 06 & 85,72 & E1; E2; E3; E4; E6; E7 \\
\hline Ansiedade moderada & 01 & 14,28 & E5 \\
\hline Ansiedade severa ou intensa & & & - \\
\hline Total & 07 & 100 &
\end{tabular}

Fonte: dados da pesquisa, 2016.

Em pesquisa de Braga; Carvalho; Binder (2010) apresentaram a prevalência dos transtornos mentais comuns (TMC) em profissionais da rede básica de saúde, apontando a presença de algum desses transtornos em $48 \%$ dos enfermeiros estudados, destacando fatores relacionados a ansiedade responsáveis por tal acometimento.

A ansiedade é possuidora de uma grande variedade de sintomas fisiológicos e de sintomas psicológicos; diversas teorias surgiram para explicá-la, tanto teorias que dão ênfase à mente, as que se associam à psicossomática, quanto àquelas que enfatizam o sistema biológico (BORINE, 2011).

De acordo com o CID 10 (2008), a ansiedade caracteriza-se na classificação de doenças como F41. As manifestações de ansiedade não estão restritas a qualquer situação ambiental em particular, sendo caracterizadas por sintomas depressivos, obsessivos e com alguns elementos de ansiedade fóbica. A ansiedade é gerada perante várias situações, com acumulo de problemas, horários a cumprir, lidar com doenças entre outras situações.

Portanto, nesse cenário de debates e estudos sobre processo de trabalho, Christovam, Porto e Oliveira (2012), buscam a construção de um conceito, denominado de gerência do cuidado, o qual está relacionado ao cuidado do enfermeiro e à própria enfermagem. Esse conceito tenta aproximar os principais processos de trabalho executados pelo enfermeiro, isto é, o gerenciar e o cuidar.

Os cuidados de enfermagem exigem profissionais preparados técnica e eticamente, comprometidos com a humanização na assistência a saúde, uma vez que lidam com vidas humanas. Os profissionais se deparam com inúmeras limitações, inerentes a própria condição de ser humano, bem como com as condições e organização do trabalho, podendo levar o cuidador a adoecer, em razão da intensa ansiedade e estresse relacionado ao processo de trabalho(BELANCIERI; KAHHALE, 2011, p. 121-128).

"É fundamental que os profissionais da área de saúde do trabalhador incentivem e 
busquem soluções viáveis para a implementação de ações de promoção da saúde e da adoção de medidas preventivas de doenças"(RIBEIRO et al, 2012, p.496).

\section{PERFIL DOS ENFERMEIROS}

Em relação ao perfil dos Enfermeiros atuantes na ESF, 100\% são do sexo feminino; $71,43 \%(05)$ encontram-se na faixa etária de 30 a 40 anos; $14,28 \%(01)$ possui 25 anos e $14,28 \%$ (01) possui 51 anos. O estado civil dos enfermeiros caracterizou-se em $71,43 \%(05)$ casados; $14,28 \%(01)$ solteiros e 14,28\%(01) união estável.

A característica histórica da enfermagem de ser exercida principalmente pelas mulheres esta ligada à própria essência da profissão: o cuidado. $O$ ato de cuidar sempre foi historicamente associado à figura feminina(FERNANDES et al, 2012).

Em relação ao tempo de trabalho como enfermeiro foi bem variado: $42,86 \%(03)$ de 01 a 10 anos; $42,86 \%(03)$ de 10 a 20 anos e $14,28 \%(01)$ de 20 a 30 anos.O tempo de trabalho especificamente na ESF foi até 20 anos: $42,86 \%$ (03) de 01 a 10 anos e $57,14 \%(04)$ de 10 a 20 anos.

Em pesquisa de Dilelio et al (2012) indicou que o maior tempo de trabalho na atenção básica pode estar associado ao aumento do desgaste físico e emocional do trabalhador.

Os enfermeiros E3; E6 e E7 possuem especialização na área de Saúde Coletiva e Saúde Pública; além de Obstetrícia (E6; E7) e gerenciamento em UBS (E7). O enfermeiro E1 possui especialização em Saúde Mental e o enfermeiro E2 em Acupuntura. Os enfermeiros E4 e E5 não possuem especialização.

É de suma importância, que o profissional de enfermagem se atualize e especialize em novos métodos de trabalhos, para um melhor aprimoramento e qualificação do cuidado. As ações de educação continuada são determinantes para a qualificação profissional e para a qualidade da assistência prestada ao usuário do serviço, constituindo-se como uma "estratégia institucional para a valorização do trabalhador" (KESSLER; KRUG, 2012, p.53).

Em relação a outros vínculos profissionais os enfermeiros E2; E6 e E7 possuem dupla jornada de trabalho:

\footnotetext{
"E2- Sim, Consultório de Acupuntura";

"E6- Sim, Farmácia e Docência";

"E7- Sim, Docência".
}

A rotina de trabalho na área da saúde, de certa forma, é desgastante por se tratar de trabalhar diretamente com pessoas e com problemas de saúde. O fato de um profissional apresentar mais de um emprego, apesar de, às vezes, ser realizado por necessidade traz a preocupação com o cuidado com a sua própria saúde, correndo o risco de poder apresentar desgaste físico e emocional. Isso não descarta a possibilidade de quem apresenta apenas um emprego, não correr o mesmo risco devido aos mesmos fatores 
citados relacionados a ansiedade.

Para os profissionais de enfermagem, a implantação da ESF significa "repensar as práticas, valores e conhecimentos, pois se amplia a complexidade das ações a serem desenvolvidas e aumenta-se os limites e possibilidades de atuação, o que requer novas habilidades" (FERNANDES et al, 2012, p.405).

\section{CATEGORIA 1 - OS FATORES ASSOCIADOS À OCORRÊNCIA DE ANSIEDADE NOS ENFERMEIROS DA ESF}

O excesso de trabalho é o maior fator causal da ansiedade, segundo os profissionais E1 a E5, além da dupla jornada de trabalho (E3 e E5).

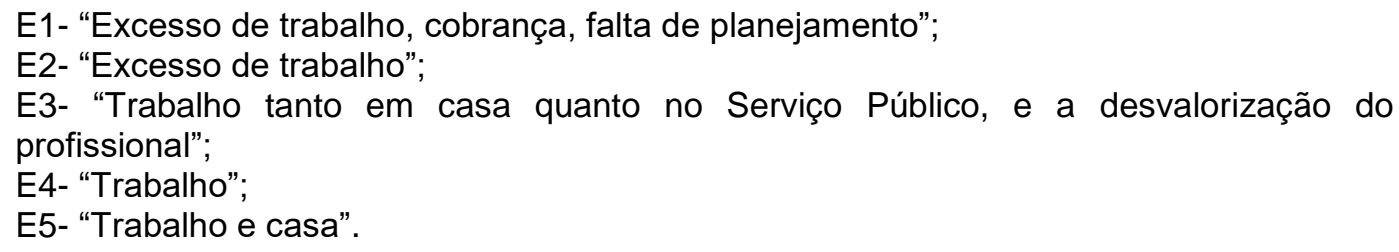

O excesso de trabalho é uma situação do cotidiano da maioria dos indivíduos podendo se tornar um fator gerador da ansiedade e adoecimento do trabalhador.

Os profissionais que cuidam sofrem uma intensa sobrecarga emocional, sendo muitas vezes negligenciada a atenção que eles conferem a si próprios. Estes profissionais são formados e especializados para o cuidado do outro, no entanto, precisam receber a mesma atenção com a própria saúde. Neste aspecto ressalta-se a importância da manutenção da qualidade de vida entre os profissionais da saúde, uma vez que os fatores psicossociais ocupacionais podem influenciar ou agravar sintomas, descaracterizando-se como uma atividade de realização pessoal e prazer, para se tornar uma via de somatização de doenças (FRONZA; TEIXEIRA, 2010).

Desta forma, a ansiedade encontra espaço para se manifestar utilizando-se de algumas atividades oportunistas, tais como, maior carga horária de trabalho, adversidades da dor e do sofrimento, o cansaço mental e físico, as situações alheias relacionadas ao estresse e as restrições organizacionais do ambiente de trabalho, gerando assim um desgaste físico e mental, prejudicando o profissional não só individualmente, tal como em seu meio social.

Considera-se que os transtornos de humor percebidos em enfermeiros podem estar associados à sobrecarga de trabalho, habilidades técnicas limitadas, gestão de conflitos e falta de apoio social no trabalho(FREITAS et al, 2014).

A falta de valorização profissional (E3; E6) e a pressão exercida no processo de trabalho na $E S F(E 6)$ foram citadas como fatores relacionados a ansiedade:

E3- "[...] desvalorização do profissional"; 
E6- "Pressão no trabalho e falta de valorização";

Um fator contributivo para insatisfação no trabalho apresentada em pesquisa de Kessler; Krug (2012) caracterizou-se pela falta de reconhecimento e valorização das atividades desempenhadas, tanto por colegas da equipe de trabalho, quanto por gestores das instituições

O trabalhador de enfermagem está exposto a diferentes estressores ocupacionais que afetam diretamente o seu bem estar, como a longa jornada de trabalho, o número insuficiente de pessoal, a falta de reconhecimento profissional, a alta exposição do profissional a riscos, o contato constante com o sofrimento e a dor (BENEVIDES-PEREIRA, 2003).

A preocupação, a agitação no cotidiano do trabalho e a dificuldade para o relaxamento foi relatado pelo enfermeiro E7 como causa para ansiedade: E7"Preocupação, fadiga, incapacidade para relaxar, agitação, etc.".

As manifestações de ansiedade ocorrem de diferentes formas, com sintomas psicológicos, como apreensão, desconforto, medos diversos e também com sintomas físicos, como taquicardia, aumento da frequência respiratória, alterações de pressão arterial etc. (ABRATA, 2011).

O estudo de Braga; Carvalho; Binder (2010) revelou que as condições de trabalho na atenção básica constituem fator contributivo ao adoecimento dos trabalhadores.

Os trabalhadores da saúde ficam expostos as exigências inerentes à atenção integral à saúde; situações geradas pela desigualdade social, assim como pelas deficiências da rede de serviços. Para atuar na atenção básica os profissionais necessitam desenvolver múltiplas habilidades e incorporar tecnologias complexas para superar os desafios do cotidiano do trabalho. A precarização do trabalho na área de saúde gera medo, isolamento e submissão dos trabalhadores. A falta de reconhecimento profissional, aliada à falta de discussão sobre planos de cargos e salários para os trabalhadores do Sistema Único de Saúde (SUS) e ao arrocho salarial no âmbito do sistema de saúde, dentre outros, são reveladores dessa precarização do trabalho na atenção básica(BRAGA; CARVALHO; BINDER, 2010).

\section{CATEGORIA 2 - O CUIDADO DO CUIDADOR}

Quando os enfermeiros foram questionados sobre como se cuidam atualmente relataram: atividades físicas (E3; E4; E6; E7); alimentação balanceada (E3; E6; E7); além de atividades de lazer como pescar, viajar (E2) e costurar (E7):

"E1- Sim";

"E2- Saio para atividades anti estresse como pescar e viajar";

"E3- Alimentação, exercícios e caminhadas";

"E4- Atividade física ao ar livre e caminhadas";

"E6- Alimentação saudável, boa ingesta hídrica e exercícios físicos"; 
"E7- Controle na dieta, atividades físicas, costuras para relaxar".

A ansiedade pode ser controlada com a adoção de novos hábitos de vida, ou seja, ofertar-se uma qualidade de vida melhor, onde faça parte de seu cotidiano atividades voltados para o bem estar físico, mental, psíquico, espiritual e social, partindo do princípio da prevenção.

A qualidade de vida no trabalho é a capacidade de administrar o conjunto das ações, incluindo diagnóstico, implantação de melhoria e inovações gerenciais, tecnológicas e estruturais no ambiente de trabalho alinhada e construída na cultura organizacional com prioridade absoluta para o bem-estar das pessoas da organização (FRANÇA, 2007, p.167.).

Em pesquisa de Kessler; Krug (2012) as práticas utilizadas no enfrentamento do sofrimento adotadas no trabalho pelos profissionais da atenção básica, o lazer traduzido por atividades físicas, viagens e festividades, foi apontado como uma alternativa para vencer a ansiedade.

Destaca-se no relato do Enfermeiro E5 a falta de cuidado de si: E5- "Não estou me cuidando".

O autocuidado é um processo cognitivo, afetivo e comportamental no qual o indivíduo assume a responsabilidade por sua própria vida, conquistando integridade nas relações consigo e com o mundo em que está inserido (SOUZA; SALOMON; LIMA, 2014, p.290).

O autocuidado é a realização de ações dirigidas a si mesmo ou ao ambiente a fim de regular o próprio funcionamento de acordo com seus interesses na vida, funcionamento integrado e bem-estar. As ações de autocuidado constituem a prática de atividades que os indivíduos desempenham de forma deliberada em seu próprio benefício com o propósito de manter a vida, a saúde e o bem-estar(BUD et al, 2006). A tarefa humana é construir o autocontrole e a moderação, é o caminho do meio, "modo de ser do trabalhador" e o "modo de ser do cuidador", pois o autocuidado não convive nem com o excesso nem com a carência, sendo o equilíbrio entre eles (BOFF, 2008).

O transtorno de ansiedade quando não tratado, dificilmente terá melhora espontânea e provavelmente irá cronificar ou ocasionar outros transtornos psiquiátricos, afetando atividades diárias e laborais, relacionamentos interpessoais. Quanto mais cedo diagnosticados os transtornos de ansiedade, avaliados e devidamente tratados, melhores os prognósticos e menores os prejuízos para o indivíduo (DESOUSA et al, 2013).

O cuidar é o objeto de trabalho dos profissionais de enfermagem e sua prática impõe uma rotina carregada de responsabilidades e tensões. Nesse contexto, é essencial "cuidar do cuidador" que estão submetidos aos "mais diversos ambientes com prolongadas jornadas de trabalho, número limitado de profissionais e desgaste psicoemocional nas tarefas realizadas"(FEITOSA et al, 2014, p.1).

\section{CATEGORIA 3 - ESTRATÉGIAS DE ENFRENTAMENTO NECESSÁRIAS PARA A}




\section{MELHORIA DA QUALIDADE DE VIDA DO ENFERMEIRO}

As estratégias de enfrentamento para a melhoria da qualidade de vida elencadas pelos enfermeiros foram principalmente voltadas à diminuição da tensão e ansiedade (E5; $\mathrm{E} 6)$; com a realização de atividades físicas (E4; E6); exercícios de respiração (E1); meditação (E6); diminuição da auto cobrança (E1); organização das atividades (E2); além de atividades de lazer (E3; E7).

\footnotetext{
E1- "Aprender a respirar e não se cobrar tanto";

E2- "Organização das atividades diárias";

E3- "Sair e fazer o que gosto";

E4- "Mudança de hábitos como mais tempo para fazer exercícios físicos";

E5- "Diminuir a tensão e humor ansioso";

E6- "Melhorar a maneira de lidar com a tensão do dia a dia com exercícios físicos e meditação";

E7- "Ler um livro que não tem haver com trabalho, assistir um filme e ficar com a família".
}

Conforme a Organização Mundial de Saúde as pessoas que são diagnosticadas com transtorno de ansiedade precocemente devem procurar métodos de relaxamento para minimizar suas tensões e medos (SOUZA et al, 2008).

A ansiedade é um dos comportamentos mais relacionados com as doenças que afeta o bem estar psicológico e/ou físico. As terapias alternativas são métodos atuais que vem sendo incorporados auxiliando na prevenção de inúmeras doenças que tenha origem nos fatores emocionais (SALLES; SILVA; 2012).

\section{CATEGORIA 4 - SUGESTÕES FRENTE À TEMÁTICA}

A sugestão mais requerida envolve a redução da carga horária de trabalho (E3; E4; E5; E7), além do aumento de recursos humanos (E4); aumento de salário e valorização profissional (E7):

"E3- Diminuição da carga horária de trabalho";

"E4- Diminuição da jornada de trabalho, ou aumento de profissionais de enfermagem por unidade";

"E5- Diminuição da carga horária";

"E7- Redução das horas trabalhadas, aumento de salário, valorização dos profissionais".

Em pesquisa de Kessler; Krug (2012) os trabalhadores da atenção básica ressaltaram a necessidade de diminuição da carga de trabalho e estabelecimento do piso salarial da categoria, a fim de minimizar os fatores condicionantes das doenças relacionadas ao trabalho.

Os enfermeiros E2 e E6 não ofereceram sugestões. O enfermeiro E1 enfatizou a importância de repassar às informações a gestão da Atenção Básica: E1- "Que os resultados sejam discutidos com gestor".

O ambiente de trabalho em saúde é ambíguo e permite ao mesmo tempo o cuidado 
e o não-cuidado de seus profissionais, o que depende dos elementos envolvidos na relação. Por um lado, a estrutura e organização, do ambiente não provêm os recursos necessários para que os profissionais - em especial, a equipe de enfermagem possa sentir-se cuidada; por outro, as relações humanas e interpessoais permitem que experimentem conforto, bemestar, realização e valorização no âmbito profissional e pessoal (OLINSKI, 2006).

Considera-se a necessidade de intervenções direcionadas ao cuidado aos trabalhadores, melhoria das condições de trabalho e aumento do suporte social no trabalho (BRAGA; CARVALHO; BINDER, 2010). As questões de saúde e, particularmente de saúde mental, da força de trabalho em saúde deve estar no centro da atenção de gestores da atenção básica(DILELIO et al, 2012).

Ações que contribuam no desenvolvimento da qualidade de vida dos profissionais das equipes de saúde da família são necessárias, considerando o fato de que a saúde dos enfermeiros pode influenciar fortemente na qualidade e humanização do cuidado prestado às famílias(FERNANDES et al, 2012).

"É preciso atentar para o paradoxo representado por situações de trabalho que contribuem para o adoecimento de trabalhadores cujas atividades visam à promoção e à recuperação da saúde de outrem" (BRAGA; CARVALHO; BINDER, 2010, p.1594).

\section{CONSIDERAÇÕES FINAIS}

Este estudo evidenciou que $85,72 \%$ dos enfermeiros apresentaram nível de ansiedade leve e $14,28 \% \%$ nível moderado. Os fatores associados à ocorrência de ansiedade nos enfermeiros da ESF vincularam-se principalmente ao excesso de trabalho, seguido da falta de valorização profissional; pressão exercida no processo de trabalho; preocupação e agitação no cotidiano do trabalho.

Em relação ao cuidado do cuidador os enfermeiros se cuidam com a realização de atividades físicas; alimentação e atividades de lazer. As estratégias de enfrentamento necessárias para a melhoria da qualidade de vida dos enfermeiros são voltadas à diminuição da tensão e ansiedade; realização de atividades físicas; exercícios de respiração; meditação; diminuição da auto cobrança; organização das atividades; além de atividades de lazer.

Os enfermeiros consideram necessária a redução da carga horária de trabalho; aumento de recursos humanos e remuneração salarial; maior valorização profissional para a melhoria da qualidade de vida no trabalho. Ainda enfatizaram a importância de discutir a temática com a gestão da Atenção Básica.

O trabalho de enfermagem compõe-se da assistência e gerencia dos serviços envolvendo esforço físico, mental e emocional; tornando-se fundamental uma reorganização do trabalho e a realização de atividades voltadas para a saúde dos profissionais. Deve-se pensar também em uma promoção da própria organização com 
ações educativas sobre as possíveis formas de enfrentamento que poderiam trazer mudanças nas condições de trabalho, tornando-a mais adequada, realizando parcerias com instituições de ensino superior para o desenvolvimento de ações em educação permanente e continuada, desta forma, trabalhando o profissional não só individualmente, mas também em grupos, ofertando terapias, relaxamentos e outros.

Desse modo, percebe-se que se torna necessário a iniciativa da gestão, de discutir estratégias que possibilitem reduzir o desgaste dos profissionais de Enfermagem, até mesmo políticas públicas de saúde no campo da saúde mental do trabalhador, pois a qualidade de vida no trabalho influencia na atenção prestada e na satisfação da prática profissional, podendo evitar o adoecimento e a absenteísmo no trabalho.

\section{REFERÊNCIAS}

ABRATA. Associação Brasileira de Familiares, Amigos e Portadores de Transtornos Afetivos. Transtorno de Ansiedade- Manual informativo. São Paulo: Planmark, 2011. 7 p. Disponível em <http://www.abrata.org.br/new/OS\%202348\%20\%20Manual\%20Paciente\%20Abrata\%202\%20-\%2010-08-11.pdf> Acesso em: 3 dez. 2015.

BANDEIRA, Roberto Albuquerque. Dor pós-operatória em idosos submetidos à prostatectomiatransvesical: correlação com a ansiedade no pré-operatório. $2010.83 \mathrm{f}$. Dissertação (Mestrado em Gerontologia) - Universidade Católica de Brasília, Brasília, 2010.

BELANCIERI, Maria de Fatima; KAHHALE, Edna Maria S. Peters. A Saúde do Cuidador: possibilidades de promoção de resiliência em enfermeiros. Reme - Rev. Min. Enferm., v.15, n.1, p.121-128, jan./mar. 2011.

BENEVIDES-PEREIRA, Ana Maria T. O Estado da arte do Burnout no Brasil. Revista eletrônica InterAçãoPsy, ano 1, n. 1, p. 4-11, Ago. 2003.

BOFF, Leonardo. Saber cuidar: Ética do humano - compaixão pela terra. 14를. Petrópolis: Vozes, 2008. 199 p.

BORINE, Monica Silvia. Ansiedade, neuroticismo e suporte familiar:Evidência de validade do Inventário de Ansiedade Traço-Estado (IDATE). 2011. 123 f. Tese (Doutorado) - Programa de Pós-graduação Strictu Sensu em Psicologia, Universidade São Francisco, Itatiba. 2011.

BRAGA, Ludmila Candida de; CARVALHO, Lidia Raquel de; BINDER, Maria Cecília Pereira. Condições de trabalho e transtornos mentais comuns em trabalhadores da rede básica de saúde de Botucatu (SP). Ciênc. saúde coletiva, Rio de Janeiro, v. 15, supl. 1, p. 1585-1596, Jun 2010. Disponível em: <http://www.scielo.br/scielo.php?script=sci_arttext\&pid=S1413$81232010000700070 \& \operatorname{lng}=\mathrm{en} \& \mathrm{nrm}=$ iso $>$. Acesso em: 22 maio 2016. http://dx.doi.org/10.1590/S1413-81232010000700070.

BRASIL. Conselho Nacional de Saúde. Resolução nº 466, de 12 de dezembro de 2012. 
Disponível em <http://conselho.saude.gov.br/resolucoes/2012/Reso466.pdf> Acesso 13 set. 2015.

BUB, Maria Bettina Camargo et al . A noção de cuidado de si mesmo e o conceito de autocuidado na enfermagem. Texto contexto - enferm., Florianópolis, v. 15, n. spe, p. 152-157, 2006 . Disponível em: <http://www.scielo.br/scielo.php?script=sci_arttext\&pid=S010407072006000500018\&Ing=en\&nrm=iso>. Acesso em:

05 Jun. 2016. http://dx.doi.org/10.1590/S0104-07072006000500018.

CID-10. Classificação Internacional das Doenças. Manual e Glossário. Porto Alegre: Artes Médicas, 2008.

CHRISTOVAM, Barbara Pompeu; PORTO, Isaura Setenta; OLIVEIRA, Denise Cristina de. Gerência do cuidado de enfermagem em cenários hospitalares: a construção de um conceito. Rev. esc. enferm. USP, São Paulo, v. 46, n. 3, p. 734-741, Jun. 2012 . Disponível em: <http://www.scielo.br/scielo.php?script=sci_arttext\&pid=S0080$62342012000300028 \&$ Ing $=$ en\&nrm=iso $>$. acesso em: 05 Jun. 2016. http://dx.doi.org/10.1590/S0080-62342012000300028.

CURY, Augusto. Ansiedade: como enfrentar o mal do século: a síndrome do pensamento acelerado: como e por que a humanidade adoeceu coletivamente, das crianças e adultos. 1. ed. São Paulo: Saraiva, 2014.

DESOUSA, Diogo Araújo et al . Revisão sistemática de instrumentos para avaliação de ansiedade na população brasileira. Aval. psicol., Itatiba, v. 12, n. 3, dez. 2013. Disponível em:<http://pepsic.bvsalud.org/scielo.php?script=sci_arttext\&pid=S167704712013000300015\&lng=pt\&nrm=iso>. Acesso em: 16 nov. 2015.

DILELIO, Alitéia Santiago et al . Prevalência de transtornos psiquiátricos menores em trabalhadores da atenção primária à saúde das regiões Sul e Nordeste do Brasil.Cad. Saúde Pública, Rio de Janeiro, v. 28, n. 3, p. 503-514, Mar. 2012.

Disponível em: <http://www.scielo.br/scielo.php?script=sci_arttext\&pid=S0102311X2012000300011\&lng=en\&nrm=iso >. Acesso em: 22 maio 2016. http://dx.doi.org/10.1590/S0102-311X2012000300011.

FEITOSA, Perla Guimaraes et al. Fatores de risco na prática clínica dos profissionais enfermeiros atuantes no Sistema Único de Saúde. Revista Interface - Comunicação, Saúde, Educação., Botucatu, supl. 3, 2014. Disponível em: <http://conferencias.redeunida.org.br/ocs/index.php/redeunida/RU11/paper/view/3320> Acesso em: 21 maio 2016.

FERNANDES, Janielle Silva et al . A relação dos aspectos profissionais na qualidade de vida dos enfermeiros das equipes Saúde da Família. Rev. esc. enferm. USP, São Paulo , v. 46, n. 2, p. 404-412, abr. 2012. Disponível em: $<$ http://www.scielo.br/scielo.php?script=sci_arttext\&pid=S0080$62342012000200019 \& \operatorname{lng}=e n \& n r m=i s o>$. Acesso em: 22 maio 2016. http://dx.doi.org/10.1590/S0080-62342012000200019.

FRANÇA, Ana Cristina Limongi: Práticas de Recursos Humanos - PRH: Conceitos, ferramentas e procedimentos. São Paulo: Atlas, 2007. 
FREITAS, Rodrigues et al. Impacto de um programa de atividade física sobre a ansiedade, depressão, estresse ocupacional e síndrome de Burnout dos profissionais de enfermagem no trabalho. Rev. Latino-Am. Enfermagem., v. 22, n. 2, p. 332-336, mar/abril. 2014.

FRONZA, Fernanda Cerveira Abuana Osório F; TEIXEIRA, Luzimar Raimundo. Perfil dos profissionais da saúde que trabalham em hospitais: relação entre sintomas musculoesqueléticos e qualidade de vida. Revista Brasileira de Ciências da Saúde., v.8, n.24, p.53-61, 2010.

HAMILTON, M. The assesment of anxiety states by rating.BrJ MedPsych,1959.

KESSLER, Adriane Inês; KRUG, Suzane Beatriz Frantz. Do prazer ao sofrimento no trabalho da enfermagem: o discurso dos trabalhadores. Rev. Gaúcha Enferm., Porto Alegre, v. 33, n. 1, p. 49-55, mar. 2012 . Disponível em: <http://www.scielo.br/scielo.php?script=sci_arttext\&pid=S1983$14472012000100007 \&$ Ing=en\&nrm=iso $>$. Acesso em: 22 maio 2016. http://dx.doi.org/10.1590/S1983-14472012000100007.

NEVES NETO, AR. Acupuntura e o tratamento da ansiedade: da MTC à psicologia. In: GUILHARDI, HJ (Org). Sobre comportamento e cognição. Santo André: Esetec; 2005. p. 95-116.

OLINISKI, Samantha Reikdal. A relação entre o cuidado de si dos profissionais de saúde e seu ambiente de trabalho: um enfoque nas interações humanas. 2006. 121f. Dissertação (Mestrado em Ciências da Saúde) - Universidade Federal do Paraná, Curitiba, 2006.

OLIVEIRA, Suenny Fonsêca de. Avaliação do programa saúde da família: uma análise a partir das crenças dos profissionais da equipe de saúde e da população assistida. 2006. 99 f. Dissertação (Mestrado Programa de Pós-Graduação em Psicologia Social) Universidade Federal da Paraíba, João Pessoa (PB), 2006.

RIBEIRO, Renata Perfeito et al . O adoecer pelo trabalho na enfermagem: uma revisão integrativa. Rev. esc. enferm. USP, São Paulo, v. 46, n. 2, p. 495-504, Abr. 2012 . Disponível em <http://www.scielo.br/scielo.php?script=sci_arttext\&pid=S0080$62342012000200031 \&$ Ing=en\&nrm=iso $>$. Acesso em:

04 out. 2015. http://dx.doi.org/10.1590/S0080-62342012000200031.

SALLES, Léia fortes; SILVA, Maria Júlia Paes. Efeito das essências florais em indivíduos ansiosos. Acta Paul Enferm.,São Paulo, v.25, n.2, p.238-42, 2012.

SALMOS, Roberto. Treinamento de força na saúde e quantidade de vida. São Paulo. Ed. Phorte, 2001. 208 p.

SANTOS, Viviane Euzebia P.; RADUNZ, Vera. O cuidar de si na visão de acadêmicas de enfermagem. Rev. enferm. UERJ, Rio de Janeiro, v.19, n.1, p.46-51, jan/mar. 2011.

SOUZA, Ângela Maria Alves e et al.Grupo Terapêutico com mulheres com Transtornos de Ansiedade: Avaliação pela escala de ansiedade de Hamilton. Rev. RENE, Fortaleza, v.9, n.1, p.19-26, jan/mar. 2008. 
SOUZA, Mara Cristina Bicudo de; SALOMON, Angélica Souza Chaves; LIMA, Beatriz Emiko Rodrigues. A prática do autocuidado pelo profissional docente enfermeiro. Revista Eletrônica Gestão \& Saúde, v.05, n. 02, p.290-02, 2014. 